\title{
Déplacements et rotations du sol lors de forts séismes à proximité de failles actives : apports des capteurs accélérométriques
}

\author{
Emmanuel Javelaud ${ }^{\star, a}$ \\ Département TEGG d'EDF-CEIDRE, 905 avenue du Camp de Menthe, 13097 Aix-en-Provence cedex 02, France
}

\begin{abstract}
Résumé - Lors de forts séismes ou près de failles actives, le sol subit des déplacements et rotations dont les amplitudes peuvent être importantes à la fois pendant et après le séisme. L'appréciation de ces mouvements du sol est fondamentale pour la prédiction des tsunamis, l'estimation des déformations du sol, ainsi que pour le dimensionnement des structures qui se trouvent à proximité de failles actives. Les déplacements peuvent être mesurés par différents moyens tels que topographiques ou géodésiques (InSAR, GPS), mais ces méthodes classiques se limitent essentiellement à la surface de la Terre et aux endroits où le géomètre peut accéder. Dans cet article, nous présentons la possibilité de calculer les déplacements ainsi que la rotation permanente du sol à partir d'accélérogrammes. L'intérêt d'utiliser les accéléromètres en complément des méthodes habituelles réside dans le nombre important d'instruments installés à l'échelle mondiale en des endroits très variés (à la surface, dans les structures, au fond de puits ou sur le fond marin) ainsi qu'au très faible pas d'échantillonnage des enregistrements, en général 100 à $200 \mathrm{~Hz}$. La méthode proposée est tout d'abord validée en des lieux où coexistent des accéléromètres et des mesures classiques. Cela permet de vérifier la justesse des déplacements et rotations permanentes calculés, puis d'utiliser cette méthode là où seuls les accéléromètres peuvent être installés. Des implications pour le dimensionnement des structures sont présentées.
\end{abstract}

Mots clés : Sismologie du mouvement fort / accélérogramme / déplacement / rotation

\begin{abstract}
Near-field displacement and rotations estimated from strong-motion accelerometers. During large earthquakes, tectonic movements along faults generate in their near-field important deformations, including transitory and permanent displacements, as well as tilting of the surface. An accurate knowledge of the ground motions is important for tsunami prediction, the estimation of ground strain, as well as for the design of structures. Several methods exist to quantitatively measure the ground deformation generated in the near-field of large earthquakes. They include topographic surveys and geodetic techniques (InSar, GPS), but these methods are limited to the Earth surface and to places where the surveyors have access. Here, we show the possibility to estimate coseismic displacements and permanent rotations from strong-motion accelerometers. Using accelerometers besides traditional techniques is interesting because of the large number of instruments deployed worldwide in a variety of different places (on the surface, within structures, boreholes or on the seafloor), and also because of the high sampling frequency of the records, usually 100 to $200 \mathrm{~Hz}$. The proposed method is validated in places where collocated accelerometers and externals measurements exist. It allows estimating the accuracy of the calculated displacements and rotations, then to use this method where accelerometers only can be installed. The implications for the design of structures are given.
\end{abstract}

Keywords: Strong-motion seismology / strong-motion accelerometer / displacement / rotation

\section{Introduction}

Lors de forts tremblements de terre, les mouvements tectoniques le long des failles génèrent à leur proximité

\footnotetext{
* Les travaux présentés ici ont été réalisés dans le cadre d'une thèse de Doctorat de Sismologie (Javelaud, 2013) au Tokyo Institute of Technology (Tokyo, Japon).

^Auteur correspondant : emmanuel.javelaud@edf .fr

${ }^{a}$ Précédemment ingénieur Ménard (Groupe Solétanche-Freyssinet), actuellement ingénieur EDF au département TEGG du CEIDRE.
}

d'importantes déformations incluant des déplacements transitoires du sol pendant la durée du séisme, des déplacements permanents, ainsi que des rotations du sol. Au cours des dernières décennies, des tremblements de terre particulièrement forts se sont produits en plusieurs régions du monde, et ont eu un impact significatif sur les populations des zones concernées. Parmi eux peuvent être cités les tremblements de terre de Mexico City (Mexique, 1985), Loma Prieta (États-Unis, 1989), Northridge (États-Unis, 1994) et Hyogoken-Nanbu (Kobe, Japon, 1995). De nombreux autres forts tremblements de terre se sont produits depuis, dont les séismes de Western Tottori 
$\left(\mathrm{M}_{\mathrm{J}}\right.$ 7,3 ; Japon, 2000), de Niigata-ken Chuetsu $\left(\mathrm{M}_{\mathrm{w}} 6,6\right.$; Japon, 2004) et de Niigata-ken Chuetsu-Oki $\left(\mathrm{M}_{\mathrm{w}} 6,8\right.$; Japon, 2007) dont les enregistrements sont utilisés dans cet article.

Une connaissance précise du déplacement du sol en zone épicentrale lors des forts séismes est nécessaire pour plusieurs applications pratiques :

- pour la prédiction des tsunamis.

Les tsunamis sont le plus souvent générés par la déformation du fond marin liée aux séismes (Dotsenko et Soloviev, 1995 ; Levin et Nosov, 2008). Le principe de la génération des tsunamis est présenté en Figure 1 ;

- pour estimer la déformation du sol.

La connaissance du déplacement du sol à différentes profondeurs est également nécessaire pour estimer la déformation du sol ainsi que pour le dimensionnement des canalisations. Lors des séismes de Hyogoken-Nanbu (1995) et Niigata-ken Chuetsu (2004), Ohtake (2006) a observé des dommages au niveau de canalisations d'adduction d'eau, en zone non liquéfiée, alors que le facteur de sécurité déterminé suivant les codes en vigueur (JSCE, 2000) était supérieur à 2. Ses travaux ont montré que les déplacements du sol en zone épicentrale ont été supérieurs à ceux calculés conformément aux codes de dimensionnement, parce que le déplacement réel au niveau du substratum géotechnique était supérieur à celui des codes. La Figure 2 montre que le déplacement à la surface est la somme du déplacement permanent du substratum sismique et du déplacement relatif dynamique dans les matériaux sédimentaires superficiels de moindres caractéristiques mécaniques ;

- pour le dimensionnement des structures et des fondations profondes.

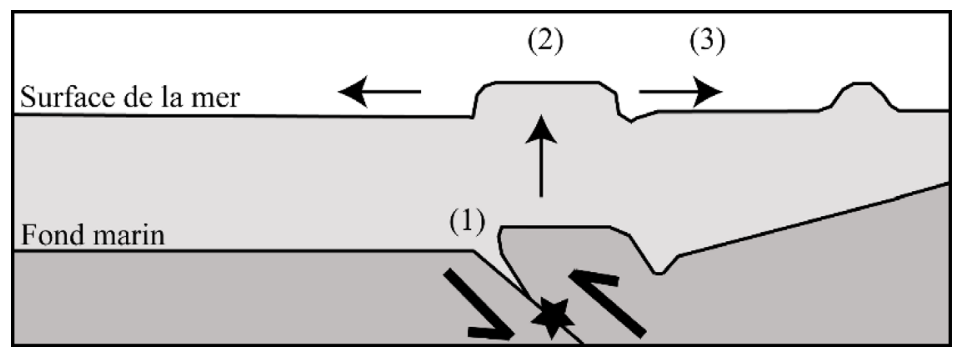

Fig. 1. Schéma de principe décrivant la génération d'un tsunami. Le mouvement brutal du fond marin à proximité de failles lors de leur jeu (1) transmet la déformation à la colonne d'eau sus-jacente et jusqu'à la surface (2). Puis, l'eau déplacée s'éloigne (3) de la zone source sous la forme d'une vague qui se propage jusqu'aux côtes. Modifié d'après IOC (2013).

Fig. 1. Principle of tsunami generation. The permanent deformation of the sea floor (1) leads to the displacement of a huge volume of water (2) that spreads out of the source area (3). Modified from IOC (2013).

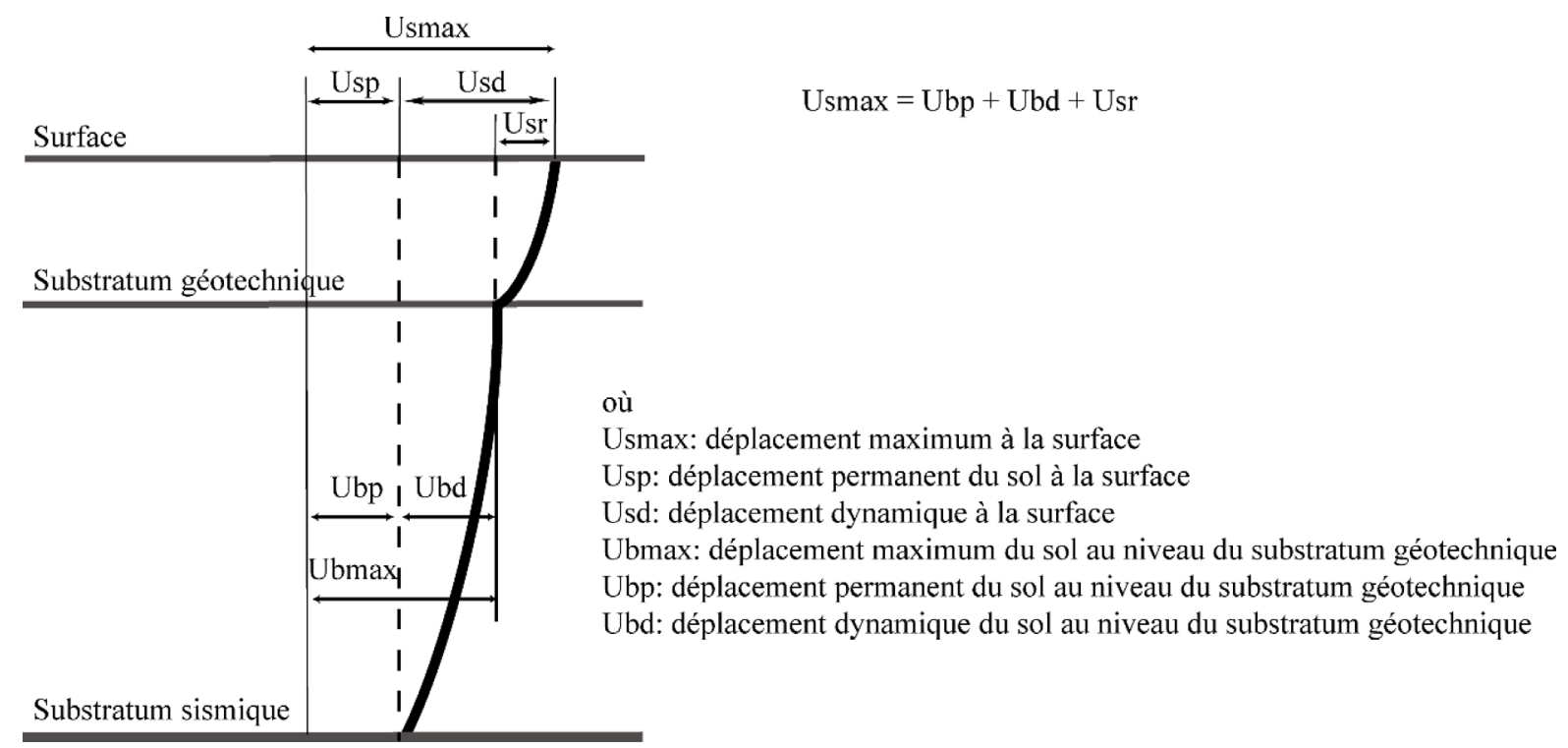

Fig. 2. Schéma de principe montrant la déformation du sol en zone épicentrale lors de forts séismes. Le déplacement à la surface est la somme du déplacement permanent du sol au niveau du substratum sismique et du déplacement relatif dynamique dans les matériaux superficiels sédimentaire de moindres caractéristiques mécaniques. Redessiné d'après Ohtake (2006).

Fig. 2. Characteristics of the ground deformation in the near-field. The displacement on the surface is the sum of the permanent ground displacement at the seismic bedrock and of the relative dynamic displacement within the superficial soft sedimentary materials. Redrawn from Ohtake (2006). 
Par ailleurs, une connaissance précise du déplacement du sol est nécessaire pour le dimensionnement des structures et des fondations profondes, en particulier pour les structures situées à proximité de failles actives où les déplacements du sol transitoires puis permanents doivent être pris en compte (e.g. Park et al., 2004).

Cet article est organisé de la manière suivante. Une première partie (Sect. 2) détaille les méthodes existantes pour mesurer le déplacement du sol.

Une deuxième partie (Sect. 3) rapelle les méthodes classiques de traitement des accélérogrammes. L'utilisation des accéléromètres connaît un regain d'intérêt en raison du développement d'instruments pour le mouvement fort ayant un bruit interne bien plus faible qu'auparavant. Une analyse détaillée des accéléromètres modernes permet d'identifier les sources de bruit de l'instrument. L'accéléromètre digital K-Net 95 présent dans les réseaux japonais K-Net et Kik-Net (NIED, 2014) est pris comme exemple. Cette analyse permet de caractériser précisément le bruit basse fréquence qui contamine l'accélération translationnelle et la rotation de l'instrument (les deux étant classiquement enregistrées par les accéléromètres).

La troisième partie (Sect. 4) décrit comment séparer l'accélération translationnelle de la rotation résiduelle dans le domaine temporel. Le déplacement translationnel est obtenu par double intégration de l'accélération translationnelle.

La méthode est ensuite validée en quatrième partie (Sect. 5) par comparaison des déplacements ainsi obtenus avec des enregistrements de stations GPS $1-\mathrm{Hz}$, et des rotations permanentes avec des mesures externes.

La cinquième partie (Sect. 6) présente des implications pour le dimensionnement des structures.

\section{Méthodes existantes pour mesurer le déplacement}

Les déplacements du sol peuvent être mesurés par différents moyens tels que topographiques ou satellitaires (nécessitant ou pas l'installation de capteurs à la surface de la Terre), et calculés à partir de vélocimètres, ainsi qu'à partir d'accéléromètres conçus pour le mouvement fort. Le Tableau 1

Tableau 1. Comparaison des différentes méthodes disponibles pour obtenir le déplacement du sol, en termes de fréquence d'échantillonnage et de localisation possible des mesures.

Table 1. Comparison of the different methods available to capture the ground displacement, in terms of their best sampling frequency as well as possible localization of the measurements.

\begin{tabular}{lll}
\hline Méthodes & $\begin{array}{l}\text { Fréquence } \\
\text { d'échantillonnage }\end{array}$ & Localisation \\
\hline $\begin{array}{l}\text { Relevé } \\
\text { topographique }\end{array}$ & Avant/après le séisme & $\begin{array}{l}\text { Limité aux endroits } \\
\text { accessibles }\end{array}$ \\
InSAR & Avant/après le séisme & $\begin{array}{l}\text { À la surface } \\
\text { GPS }\end{array}$ \\
Vélocimètres & $1 \mathrm{~Hz}$ & $\begin{array}{l}\text { À la surface } \\
\text { Grottes pour le réseau } \\
\text { F-Net (Japon) } \\
\text { N'importe où }\end{array}$ \\
\hline
\end{tabular}

précise pour chaque méthode la meilleure fréquence d'échantillonnage possible ainsi que les limitations en termes de localisation. Elles sont détaillées ci-dessous :

- les relevés topographiques.

Les relevés topographiques permettent d'avoir des informations précises sur le déplacement permanent de points de références dont les coordonnées sont connues avant le séisme. Ils se limitent aux endroits physiquement accessibles à l'homme pour les mesures ;

- les méthodes géodésiques.

Les méthodes géodésiques sont restreintes quant à elles à la surface de la Terre. Elles donnent des informations sur les déplacements permanents pour la méthode InSAR (Massonnet et al., 1993), et sur les déplacements transitoires et permanents à des fréquences d'échantillonnage allant jusqu'au hertz pour les récepteurs GPS préinstallés avant le séisme dans la zone d'étude (Bock et al., 1993).

En complément peuvent également être utilisés des sismomètres faits pour enregistrer le mouvement de translation du sol :

- les vélocimètres.

Parmi eux se trouvent les vélocimètres sensibles, comme ceux du réseau F-Net (NIED, 2014) au Japon. Ils permettent théoriquement de calculer le déplacement transitoire et permanent de l'instrument par intégration du signal enregistré. Cependant, les vélocimètres enregistrent non seulement la vitesse du sol mais également la rotation de l'instrument. Ils présentent de plus des phénomènes de saturation lors de fortes sollicitations ;

- les accéléromètres conçus pour mesurer le mouvement fort du sol.

Enfin, les accéléromètres conçus pour la mesure du mouvement fort peuvent être utilisés pour calculer le déplacement du capteur par double intégration du signal enregistré. Comme les vélocimètres, ils sont sensibles à la rotation de l'instrument pendant le séisme, et présentent des limitations instrumentales.

\section{Potentialité des accéléromètres}

L'utilisation d'accéléromètres faits pour enregistrer le mouvement fort du sol est très intéressante pour déterminer le déplacement du sol en raison :

- du nombre important d'instruments installés à l'échelle mondiale ;

- de leur répartition : en surface, dans les structures ou au fond de puits, mais également sur le plancher océanique ;

- de la facilité d'accès aux données, souvent libre et gratuit ;

- de leur haute fréquence d'échantillonnage, habituellement 100 à $200 \mathrm{~Hz}$.

\subsection{Limitations classiques des accéléromètres}

Théoriquement, la vitesse du sol et son déplacement de translation peuvent être calculés par simple et double intégration des accélérogrammes. En pratique, les vitesses et déplacements ainsi calculés sont irréalistes (e.g. Fig. 3), avec à la fin du séisme une vitesse du sol non nulle et un déplacement 
non stabilisé. Cela n'a pas de réalité physique. Ces dérives sont dues à du bruit longue période qui contamine l'enregistrement de l'accélération translationnelle et qui produit les fortes divergences observées après double intégration.

L'origine de ce bruit a été attribuée essentiellement à des imperfections instrumentales, à du bruit électronique interne au capteur, et à l'enregistrement de la rotation de l'instrument simultanément à celui de l'accélération translationnelle (e.g. Boore et Bommer, 2005 ; Graizer, 2010 ; Kinoshita, 1998 ; Trifunac et al., 1973).

Des méthodes ont été proposées pour supprimer ce bruit longue période. Elles incluent couramment l'utilisation de filtres (Boore et Bommer, 2005; Trifunac, 1971) ou la soustraction de fonctions simples ou plus complexes des enregistrements (Boore et Bommer, 2005 ; Iwan et al., 1985 ; McComb et al., 1943). La Figure 4 (a) montre l'accélération enregistrée pendant le séisme de Chi-Chi en $1999\left(M_{w} 7,6\right)$ à
Taiwan par la station TCU129 (composante EW), et la Figure 4 (b) la vitesse obtenue par simple intégration. Fondée sur l'observation des dérives de la vitesse, la Figure 4 (b et c) présente plusieurs options possibles pour corriger l'accélérogramme (Boore, 2001). Typiquement, une vitesse qui croît linéairement avec le temps est due à l'ajout d'une constante dans l'accélération. Les corrections apportées conduisent ainsi à la soustraction de créneaux ou d'autres fonctions plus complexes de l'accélérogramme, mais présentent la difficulté de la sélection de la correction donnant un déplacement réaliste.

Aujourd'hui, les dernières générations d'accéléromètres développés pour la mesure du mouvement fort ont un bruit interne bien plus faible qu'auparavant. Il est désormais possible de déterminer quels sont les bruits élémentaires qui contaminent l'accélération translationnelle, et donc de proposer des méthodes d'analyses originales spécifiques pour chaque
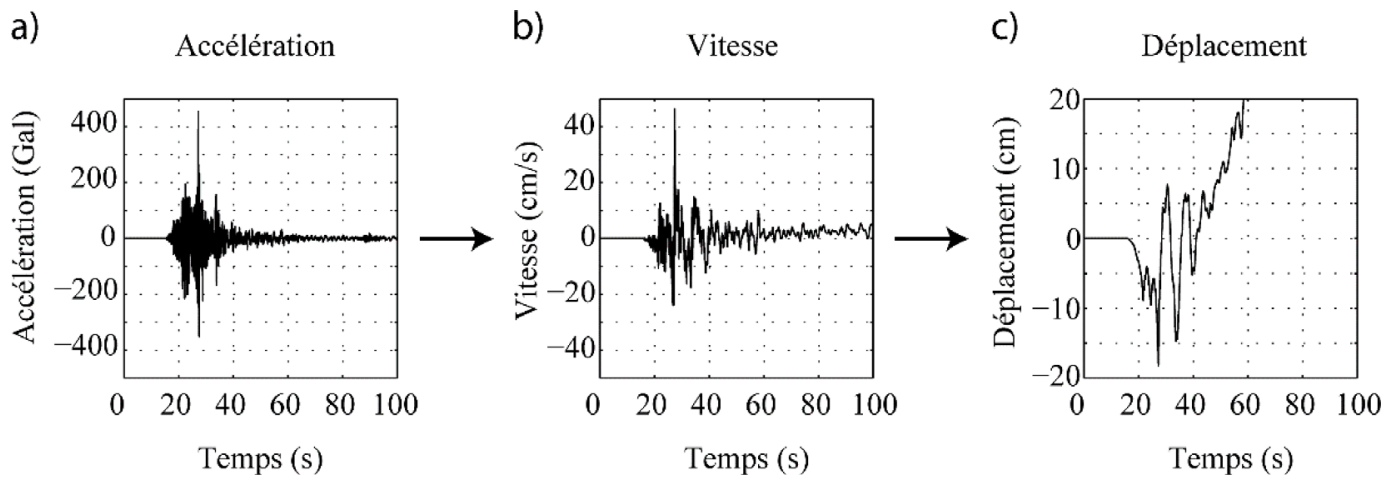

Fig. 3. (a) Accélération enregistrée pendant le séisme de Niigata-ken Chuetsu-Oki en 2007 au Japon par la station NIG019 (composante EW) du réseau K-NET. $(b, c)$ Vitesse et déplacements obtenus par simple et double intégration de l'accélération, après soustraction de l'accélération moyenne précédant le séisme.

Fig. 3. (a) Acceleration time history recorded during the 2007 Niigata-ken Chuetsu-Oki earthquake (Japan) at K-Net NIG019 station (EW component). (b, c) Velocity and displacement time series obtained by simple and double integration of the acceleration record, after removal of the pre-event mean from the whole record.
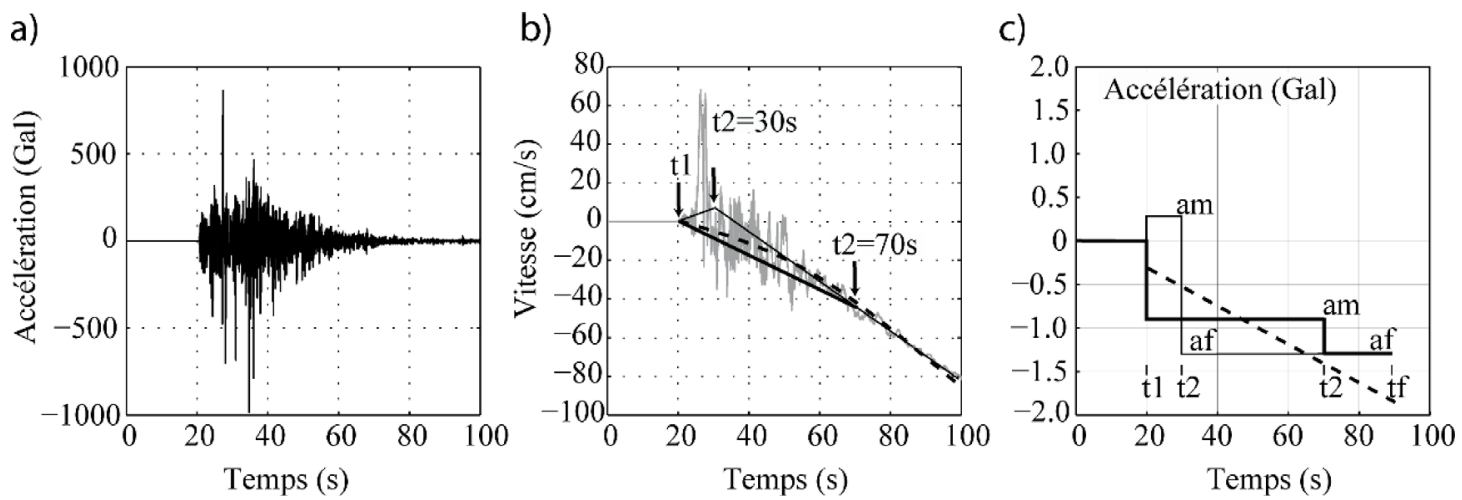

Fig. 4. (a) Accélération enregistrée pendant le séisme de Chi-Chi en 1999 (Taiwan) par la station TCU129 (composante EW). L'instrument était situé à $1,9 \mathrm{~km}$ de la faille. (b) Vitesse obtenue par simple intégration de l'accélération, après soustraction de l'accélération moyenne précédant le séisme. Boore (2001) a proposé plusieurs façon de corriger cette dérive. (c) Les dérivées des corrections définies en (b) sont les corrections qui sont appliquées aux accélérogrammes. Les figures (b et c) ont été redessinées à partir de Boore (2001) et Boore et Bommer (2005).

Fig. 4. (a) Acceleration time history recorded during the 1999 Chi-Chi earthquake (Taiwan) at station TCU129 (EW component). The instrument was situated $1.9 \mathrm{~km}$ from the surface trace of the fault. (b) Velocity time history obtained by integration of the acceleration record, after removal of the pre-event mean from the whole record. Boore (2001) proposed several processing schemes. (c) The derivatives of the lines fit to the velocity are the baseline corrections applied to the acceleration trace. Figures ( $b$ and $c$ ) are redrawn from the parameters given by Boore (2001) and Boore and Bommer (2005). 
enregistrement afin de supprimer un à un les différents types de bruit présents dans les accélérogrammes.

\subsection{Caractéristiques des accéléromètres modernes : exemple du K-Net 95}

Les enregistrements sismologiques en zone épicentrale lors de forts séismes sont réalisés par des accéléromètres conçus pour mesurer les fortes accélérations translationnelles du sol. Sur le principe, un accéléromètre numérique est conçu pour mesurer l'accélération de translation du sol et fournit à l'utilisateur l'amplitude du signal à des intervalles de temps réguliers (Fig. 5).

L'accéléromètre K-Net 95 est présent dans les réseaux japonais K-Net et Kik-Net (NIED, 2014) où plus de 2000 unités ont été installées. Une analyse détaillée des différents éléments constituant l'accéléromètre K-Net 95 (Kinoshita, 1998) permet d'évaluer le bruit longue période généré individuellement par chaque composant. Leur somme forme collectivement le bruit présent dans les enregistrements.

L'équation du capteur accélérométrique (NIED, 2000 ; Pillet et Virieux, 2007) montre que ses composantes horizontales enregistrent non seulement l'accélération translationnelle $x_{i}$ " mais également la rotation $\gamma_{\mathrm{j}}$ de l'instrument sous la forme $g$. Sin $\gamma_{\mathrm{j}}$, où $g$ est l'accélération de la gravité. De plus, pendant l'acquisition, ce signal d'entrée est modifié par la réponse du capteur qui est la somme de sa réponse théorique et de bruit électronique en $1 / f$ à basses fréquences. Les accélérogrammes sont donc systématiquement contaminés à basses fréquences par du bruit en $1 / \mathrm{f}$ (pente -1 dans un diagramme log-log) dont l'amplitude peut être estimée à partir d'enregistrements de bruit de fond. Le contenu basse fréquence des accélérogrammes est donc dominé soit par le bruit en $1 / f$, soit par la rotation permanente de l'instrument.

\section{Méthode d'analyse des accélérogrammes}

La méthode d'analyse proposée est fondée sur l'observation du contenu basse fréquence des accélérogrammes dans le domaine fréquentiel, et sur les positions relatives à basse fréquence de l'accélération translationnelle, de la rotation et du bruit interne en $1 / \mathrm{f}$. Les différentes étapes sont synthétisées sur la Figure 6.

Deux cas principaux se présentent. Dans le premier, à basse fréquence, le bruit en 1/f a une amplitude supérieure à l'accélération translationnelle et à la rotation permanente (cas 1, Sect. 4.2). Dans ce cas, il n'est pas possible d'estimer le

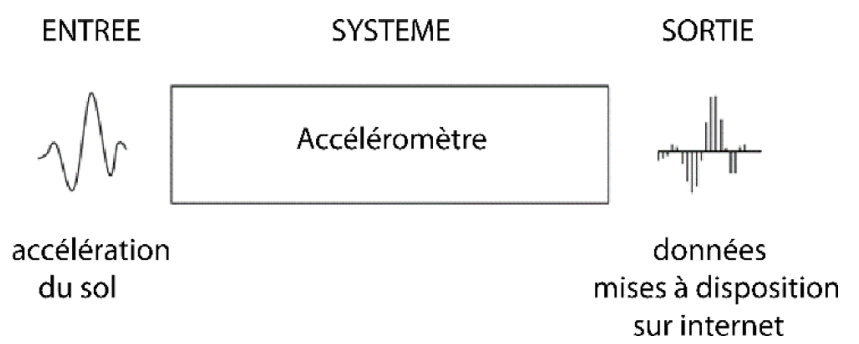

Fig. 5. Principe de l'accéléromètre mesurant le mouvement fort. Fig. 5. Schematic view of the strong-motion seismometer. déplacement translationnel, sauf si le bruit en $1 / \mathrm{f}$ a une amplitude supérieure à l'accélération translationnelle et à la rotation permanente uniquement jusqu'à des fréquences très petites. On se trouve ainsi dans le cas intermédiaire. Dans une deuxième situation, l'information relative à la rotation domine le contenu basse fréquence de l'enregistrement (cas 2, Sect. 4.3). Dans ce cas, il est possible de la soustraire de l'enregistrement dans le domaine temporel et d'estimer le déplacement translationnel.

\section{1 Étapes préparatoires}

Trois étapes préliminaires permettent de faciliter la visualisation du contenu basse fréquence des accélérogrammes, et de discuter leur contenu.

Tout d'abord, la moyenne de l'accélération sur la portion de signal enregistrée avant l'occurrence du séisme est soustraite de l'ensemble de l'accélérogramme, de façon à ce que la condition physique d'une accélération nulle avant le séisme soit respectée. Cela permet de s'affranchir des dérives très lentes de la ligne de base des accélérogrammes dues en particulier aux variations des conditions externes comme la température.

Ensuite, la transformée de Fourier de l'enregistrement est réalisée en calculant les amplitudes de Fourier à des fréquences très rapprochées de façon à avoir une visualisation détaillée du contenu fréquentiel à basses fréquences. L'ajout d'un grand nombre de zéro aux enregistrements permet le calcul de la transformée de Fourier discrète avec un pas d'échantillonnage très faible.

Enfin, l'amplitude du bruit en $1 / \mathrm{f}$ à basse fréquence est estimée pour chaque composante de chaque accéléromètre en utilisant des enregistrements de bruit de fond.

\subsection{Cas 1 : le contenu basse fréquence est dominé par le bruit en $1 / f$}

À basse fréquence, la présence de bruit en 1/f, d'amplitude supérieure à l'accélération translationnelle et à la rotation (Fig. 7), rend l'obtention d'un déplacement translationnel réaliste impossible.

Cette situation est de moins en moins fréquente avec le développement et l'installation de nouveaux instruments ayant un bruit interne en $1 / \mathrm{f}$ de plus en plus faible. Cela permet en effet d'être dans le cas intermédiaire où un déplacement réaliste est obtenu par double intégration de l'accélérogramme lorsque le bruit en 1/f domine le contenu de l'enregistrement jusqu'à de très petites fréquences uniquement.

\subsection{Cas 2 : le contenu basse fréquence est dominé par la rotation permanente}

À basse fréquence, l'information relative à la rotation domine le contenu des accélérogrammes.

La façon la plus simple de modéliser la présence de rotation permanente lors d'un séisme est d'utiliser une fonction créneau, d'amplitude $A$ et de durée $T$ (Fig. 8 (a)) jusqu'à la fin de l'enregistrement.

Les paramètres $A$ et $T$ de cette fonction créneau peuvent être déterminés de façon unique dans le domaine de Fourier (Fig. 8 (b)) : par définition, sa transformée de Fourier a une amplitude 


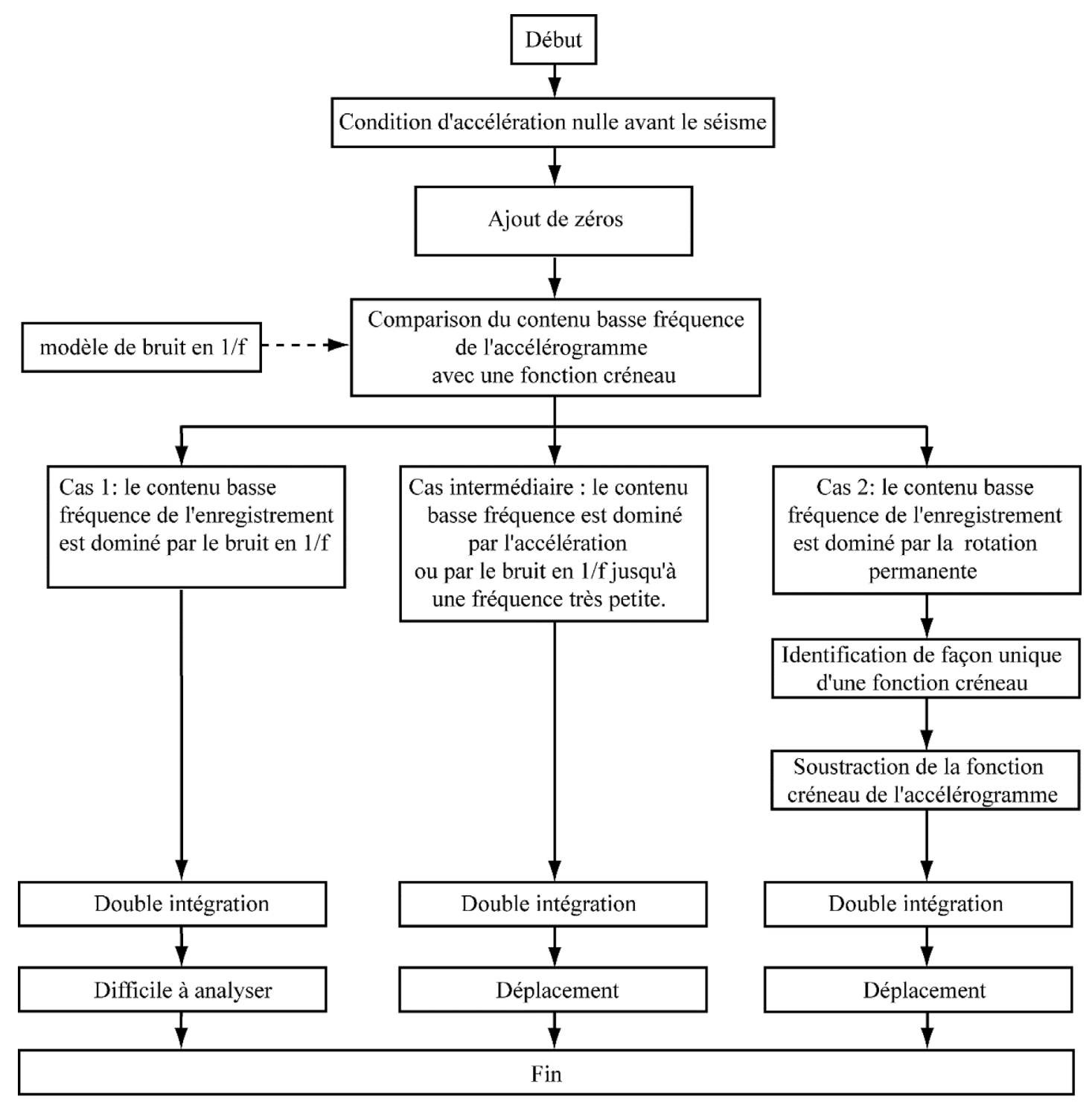

Fig. 6. Schéma décrivant les étapes de la méthode proposée.

Fig. 6. Flow chart of the proposed processing scheme.

$A . T$ à fréquence nulle, et la fréquence pour laquelle l'amplitude de Fourier est nulle pour la première fois est $1 / T$. Ainsi, les paramètres $A$ et $T$ de la fonction créneau peuvent être extraits de la transformée de Fourier de l'enregistrement.

Pendant le séisme de Niigata-ken Chuetsu-Oki en 2007, l'accéléromètre K-Net NIG019 était déjà situé dans ce qui fut la zone épicentrale du séisme. Sur la Figure 8, nous comparons l'accélérogramme et sa transformée de Fourier (c, d) avec une fonction créneau et sa transformée de Fourier (a, b). Dans le domaine de Fourier, la partie basse fréquence de l'accélérogramme (Fig. 8 (d)) est identique à une fonction créneau. Cela indique que l'accélérogramme est contaminé par une fonction créneau, dont l'amplitude $A$ et la durée $T$ peuvent être déterminées de façon unique. Dans cet exemple, nous obtenons les valeurs suivantes à partir de la transformée de Fourier de l'accélérogramme (Fig. 8 (d)) $: A \cdot T=11,7177$ Gal.s (amplitude de Fourier à fréquence nulle) et $1 / T=0,0036467 \mathrm{~s}^{-1}$ (fréquence pour laquelle l'amplitude de Fourier est nulle pour la première fois). Donc $T=1 /(1 / T)=274,2186 \mathrm{~s}, A=A \cdot T / T=0,042731 \mathrm{Gal}$ et le créneau débute au temps

$$
\begin{aligned}
\left(t_{s}\right) & =\text { duree de } \mathrm{l}^{\prime} \text { enregistrement }-T=300 \mathrm{~s}-T \\
& =25,7814 \mathrm{~s} .
\end{aligned}
$$

Sur la Figure 9 (b), la superposition dans le domaine de Fourier de l'accélérogramme et d'une fonction créneau de caractéristiques $A$ et $T$ déterminées précédemment, montre une très bonne similitude à basses fréquences.

Les étapes suivantes du traitement sont réalisées dans le domaine temporel. Une fonction créneau d'amplitude $A=0,042731 \mathrm{Gal}$ et de temps de départ $t_{s}=25,7814 \mathrm{~s}$ est soustraite de l'accélérogramme (Fig. 9 (a)). La double intégration de l'accélérogramme corrigé ne présente plus de dérive : le déplacement est constant après le séisme (Fig. 9 (d)). Le déplacement résiduel est estimé à $-3,2 \mathrm{~cm}$. 


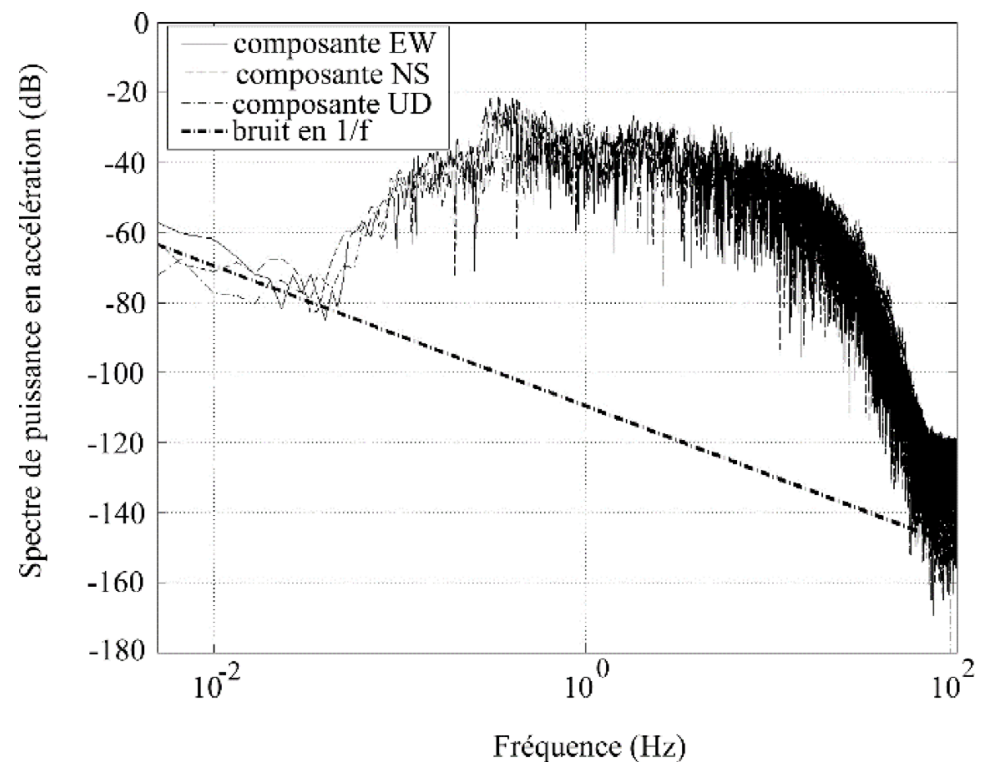

Fig. 7. Spectre de puissance calculé à partir d'un enregistrement de la station NIGH04, sismomètre en fond de trou, pendant le séisme de Niigataken Chuetsu (2004, Japon). L'utilisation du spectre de puissance permet la comparaison avec l'article original de Kinoshita. Reproduit de Javelaud et al. (2011a).

Fig. 7. Power spectrum calculated from a record at Sekikawa NIGH04 site, underground seismometer, during the 2004 Niigata-ken Chuetsu earthquake. The power spectrum representation allows comparison with Kinoshita's original papers. From Javelaud et al. (2011a).
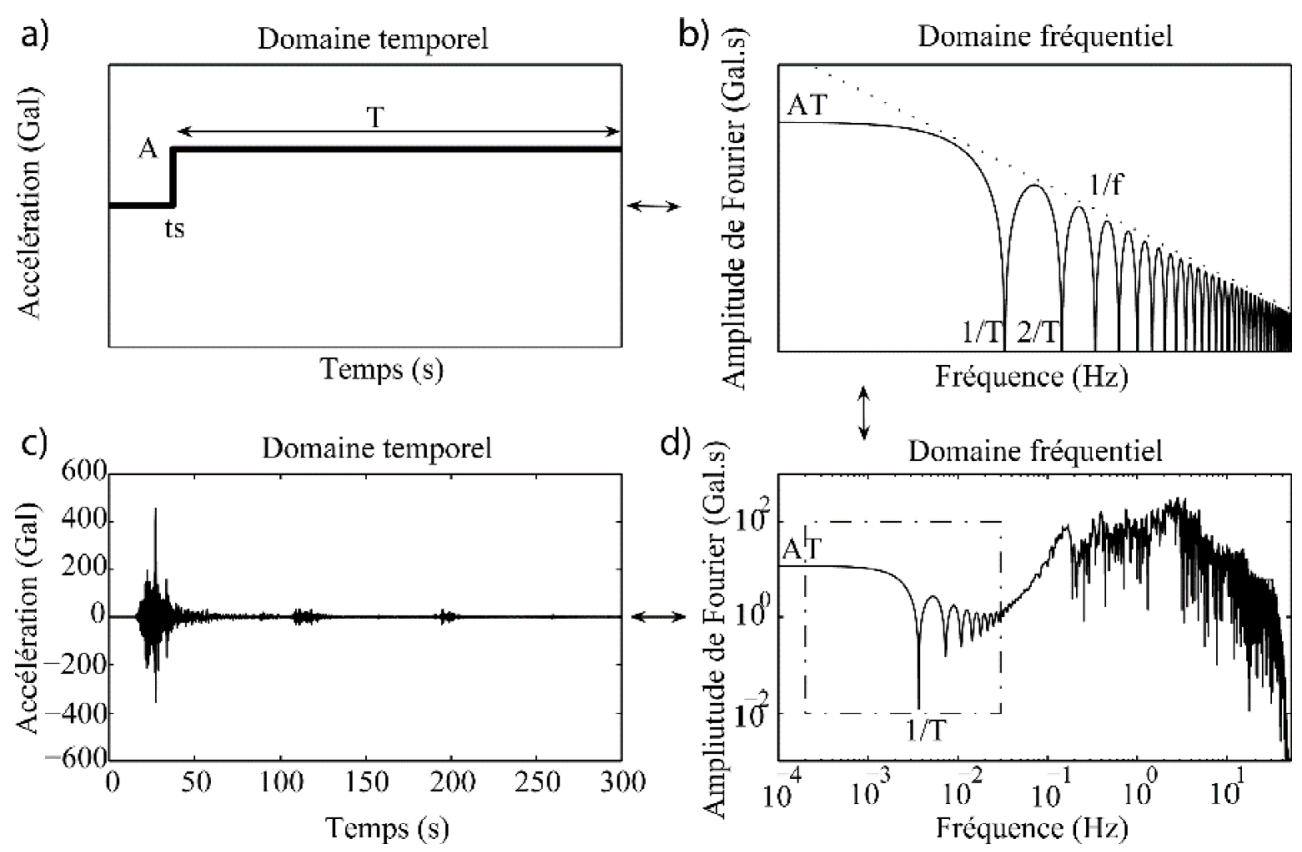

Fig. 8. Comparaison d'une fonction créneau $(a, b)$ et d'un accélérogramme $(c, d)$ dans les domaines temporels et de Fourier. Exemple de l'enregistrement du séisme de Niigata-ken Chuetsu-Oki (2007) fait par l'accéléromètre NIG019, composante EW. Reproduit de Javelaud et al. (2011a).

Fig. 8. Comparison of a step function $(a, b)$ and an acceleration record $(c, d)$ in both time and Fourier domains. Example from the 2007 Niigataken Chuetsu-Oki earthquake, NIG019 station, EW component. From Javelaud et al. (2011a). 
a)

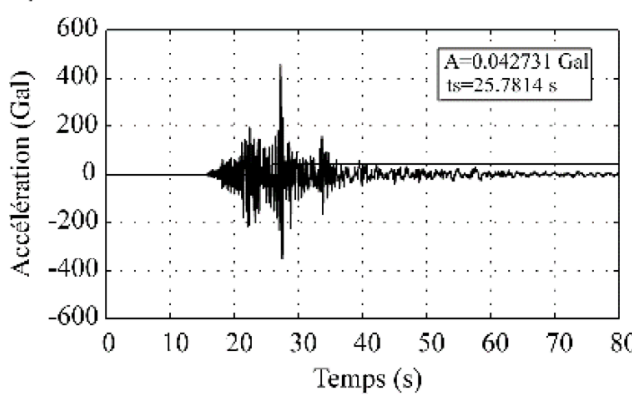

c)

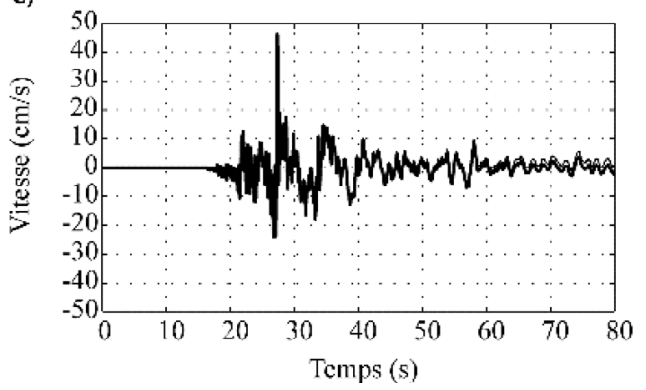

b)

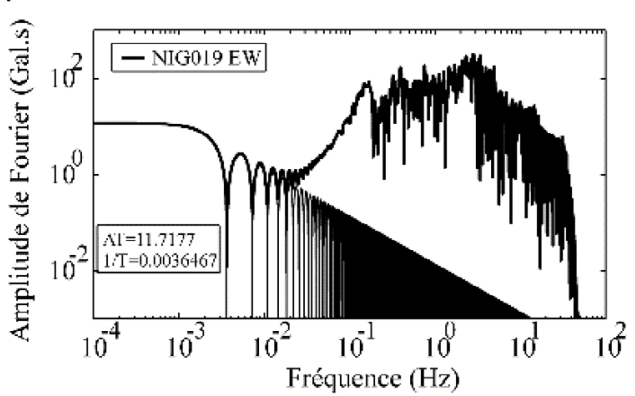

d)

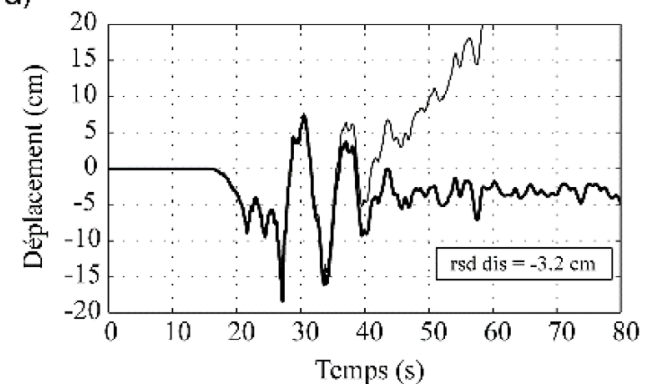

Fig. 9. (a) Superposition de l'accélérogramme et d'une fonction créneau d'amplitude A (non à l'échelle) et de temps de début $t_{s}$ (déterminé précédemment) utilisé pour corriger l'accélérogramme. (b) La superposition dans le domaine de Fourier de l'accélérogramme et du créneau d'amplitude $\mathrm{A}$ et de temps de début $t_{s}$ présente une très bonne correspondance à basse fréquence. (c, d) Vitesse et déplacement non corrigés et corrigés. Le déplacement corrigé est obtenu en soustrayant le créneau à l'accélérogramme non corrigé, avant d'intégrer deux fois l'accélérogramme corrigé. Le déplacement est stable après le séisme. Reproduit de Javelaud et al. (2011a).

Fig. 9. (a) Superposition of the acceleration record and a step function of amplitude A (not to scale) and starting time $t_{s}$ (determined above) used to correct the acceleration record. (b) The superposition in the frequency domain of the acceleration record and the step function of amplitude $A$ and starting time $t_{s}$ shows extremely good match at low frequency. $(c, d)$ Uncorrected and corrected velocity and displacement time histories. The corrected displacement history is obtained by subtracting the step function from the acceleration record, then double integrating the corrected acceleration time history. The displacement is stable after the earthquake. From Javelaud et al. (2011a).

\section{Vérifications des déplacements et rotations obtenus}

La méthodologie détaillée aux Sections 4.2 et 4.3 est appliquée à des enregistrements réalisés en zone épicentrale lors de forts séismes. La justesse des résultats obtenus en termes de déplacement et de rotation est estimée par comparaison avec des mesures externes.

\subsection{Comparaison des rotations permanentes calculées avec des données externes mesurées}

L'analyse des accéléromètres a montré qu'ils enregistrent l'accélération translationnelle ainsi que la rotation. Les constantes $A(\mathrm{Gal})$ soustraites des accélérogrammes dans le cas d'analyse 2 sont a priori des enregistrements de la rotation permanente $\gamma$ de l'instrument sous la forme $A=g$. Sin $\gamma$. La justesse des angles de rotation calculés à partir d'accélérogrammes est vérifiée en confrontant les angles $\gamma$ obtenus avec des mesures externes, ce qui est possible sur des sites exceptionnels pour lesquels la rotation a été calculée et mesurée de plusieurs façons.

Construit en 1989, le barrage de Kasho au Japon s'est trouvé à l'aplomb d'une faille aveugle active lors du séisme de Western Tottori $\left(M_{J} 7,3\right)$ en l'an 2000. Cette situation en fait un cas d'étude bien adapté. Tout d'abord en raison des accélérations très importantes mesurées pendant le séisme, avec des valeurs proches de $2 \mathrm{~g}$ enregistrées à la crête du barrage (JCOLD, 2002). Puis, en raison des mouvements importants qu'il a subis, incluant un déplacement permanent de la structure et une rotation permanente tectonique de l'ordre de $10^{-4} \mathrm{rad}$. Enfin, l'évolution régulière du champ de déformations à l'échelle de l'ouvrage et de la retenue (une zone de quelques kilomètres carrés selon la simulation numérique faite par Ohmachi et al., 2003) valide la possibilité de comparer la rotation obtenue dans cette zone par différents outils.

Pour cet ouvrage, quatre méthodes différentes permettent de mesurer et de calculer la rotation tectonique permanente qu'il a subie : l'accéléromètre situé à la base du barrage (Fig. 10), des mesures topographiques réalisées tout autour de la retenue, des simulations numériques (Ohmachi et al., 2003) ainsi qu'un pendule situé dans l'ouvrage (Fig. 10).

L'analyse des deux accélérogrammes (Fig. 11 (a, b)) enregistrés à la fondation du barrage est faite conformément à la méthode proposée à la Section 4. Dans les deux cas (Fig. 11 (c, d)), leurs amplitudes de Fourier à basse fréquence sont bien audessus du bruit en $1 / \mathrm{f}$ déterminé indépendamment à partir de mesures de bruit de fond, et sont similaires à des fonctions créneaux. Ces situations correspondent à des cas d'analyse 2 des accélérogrammes. La méthode du cas 2 est donc appliquée à chaque enregistrement, et les paramètres des fonctions créneaux sont déduits de l'observation de leur transformée de Fourier : $A=-0,06952 \mathrm{Gal}$ et $A=-0,08162 \mathrm{Gal}, t_{s}=20,84 \mathrm{~s}$ et 


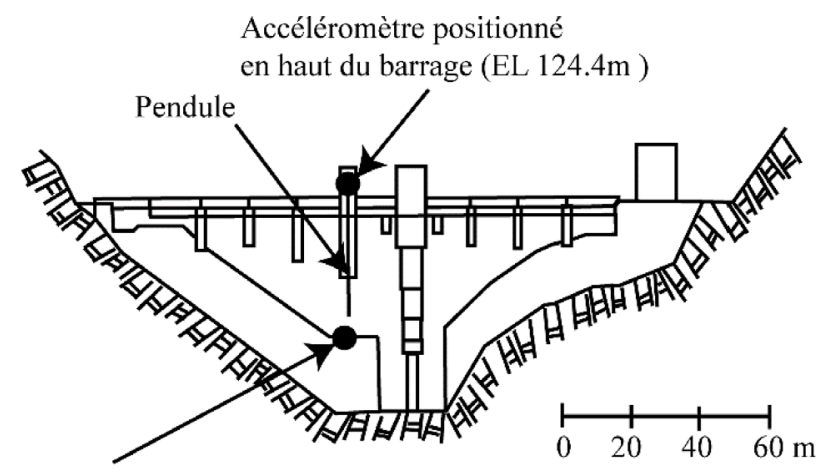

Accéléromètre situé

à la base du barrage (EL $87.0 \mathrm{~m})$

Fig. 10. Schéma du barrage de Kasho montrant l'emplacement des accéléromètres et du pendule.

Fig. 10. Schematic explanation of Kasho dam showing the location of the strong-motion seismometers and the plumb line.

$t_{s}=24,96 \mathrm{~s}$ pour les composantes EW et NS respectivement. Puis les fonctions créneaux sont soustraites de chaque accélérogramme, dans le domaine temporel.

La double intégration de ces accélérations corrigées ne présente plus de dérive (Fig. $11(\mathrm{~g}, \mathrm{~h})$ ). Les déplacements résiduels sont de $-4,0 \mathrm{~cm}$ et de $+27,4 \mathrm{~cm}$ pour les composantes $\mathrm{EW} \mathrm{et}$ NS respectivement. Le déplacement global de l'ouvrage, calculé comme la résultante des déplacements de chaque composante, est ainsi de $27,7 \mathrm{~cm}$ dans la direction $\mathrm{N} 341^{\circ}$, du même ordre de grandeur que les déplacements topographiques mesurés.

La rotation permanente $\gamma$ est calculée pour chaque composante à partir de l'amplitude $A=g \cdot \operatorname{Sin} \gamma$ de la fonction créneau soustraite de chaque composante, puis comparée aux rotations permanentes mesurées ou déterminées par les autres méthodes. Bien que les quatre différentes méthodes ne mesurent pas exactement la même chose (mesure ou calcul ponctuels, ou bien sur de plus grandes surfaces ; rotation durant le choc principal ou durant des répliques), les rotations permanentes déterminées présentent une bonne homogénéité. Elles donnent une rotation permanente de direction $\mathrm{N} 240^{\circ} \pm 30^{\circ}$ ainsi qu'un angle de rotation de $11,5 \pm 5,5 \times 10^{-5}$ rad, ce qui confirme la possibilité d'estimer des rotations permanentes réalistes à partir d'accélérogrammes.

\subsection{Comparaison des déplacements calculés avec des données externes mesurées}

La justesse des déplacements calculés par double intégration des accélérogrammes corrigés peut être vérifiée en les confrontant à des mesures externes.

La méthode décrite à la Section 4 est appliquée aux enregistrements de dix stations accélérométriques situées en zone épicentrale pendant le séisme de Niigata-ken Chuetsu-Oki (2007, Japon). La position des différents capteurs accélérométriques est indiquée sur la Figure 12. Pour chaque accélérogramme, sa transformée de Fourier, la vitesse, et le déplacement ont été calculés. Des déplacements permanents stables après le séisme ont été obtenus dans des cas d'analyse 2 (8 enregistrements) et des cas d'analyse intermédiaire (4 enregistrements). Cependant, pour des situations d'analyse 1 (5 enregistrements), des déplacements permanents stables ne sont pas toujours obtenus après le séisme, et le déplacement permanent est alors estimé.

Pour s'assurer de l'efficacité de la méthode, les déplacements calculés à partir des accélérogrammes sont comparés à ceux mesurés à proximité par des stations GPS (Fig. 12).

Pendant le séisme, la station K-Net NIG019 Ojiya et la station GPS 1-Hz Ojiya ont toutes deux enregistré l'événement. Les deux stations sont situées à $600 \mathrm{~m}$ l'une de l'autre. Pour les composantes EW et NS, le déplacement du sol pendant le séisme déterminé par les deux méthodes (Fig. 13) présente une très bonne similitude à la fois pendant la durée du séisme et une fois ce dernier terminé.

À Kashiwasaki, les déplacements calculés à partir des accélérogrammes de la station JMA sont comparés aux données GPS de la station K1 située à 3,5 km de distance. Pour la composante EW, le déplacement permanent obtenu par les deux méthodes présente une bonne similitude et une valeur de $14 \mathrm{~cm}$. Pour l'accéléromètre, l'amplitude des oscillations du déplacement pendant le séisme atteint $80 \mathrm{~cm}$, et il y a globalement une bonne correspondance entre les déplacements obtenus par les deux méthodes. Le mouvement permanent est ici l'aboutissement d'un mouvement d'amplitude beaucoup plus importante. Les différences existantes peuvent provenir du champ de déformations qui varie latéralement très rapidement et de possibles décalages dans l'orientation des instruments. En ce qui concerne la composante NS de l'accélérogramme, un pic tardif à la quarantième seconde rend la méthodologie impossible à appliquer.

À Takayanagi, les deux stations sont situées à une distance de $1,2 \mathrm{~km}$ l'une de l'autre. Les déplacements permanents calculés et mesurés sont similaires tandis que les déplacements pendant la durée du séisme présentent de fortes similitudes compte-tenu de la distance séparant les instruments.

Pour les deux autres stations Mashima et Matsunoyama, les déplacements obtenus par les deux méthodes présentent de bonnes similitudes considérant que la distance entre les instruments est de plusieurs kilomètres.

Les déplacements permanents obtenus à partir d'accéléromètres et de données GPS sont reportés sur la Figure 12. Quand les stations accélérométriques et GPS sont situées à une distance de quelques centaines de mètres l'une de l'autre, les 
a)

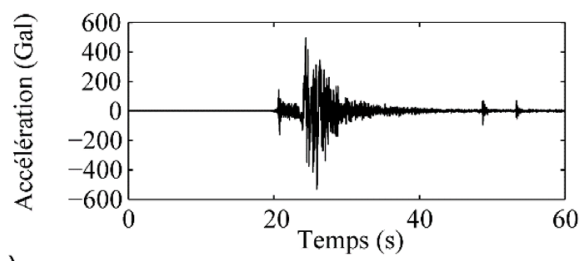

C)
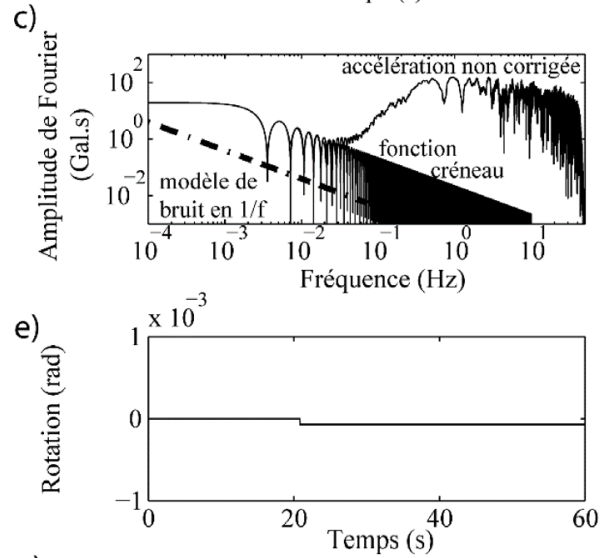

g)

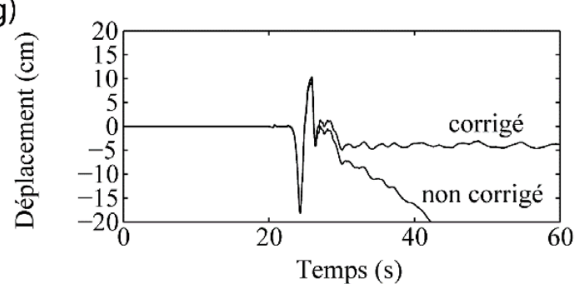

b)

Gallery d'inspection basse, composante NS

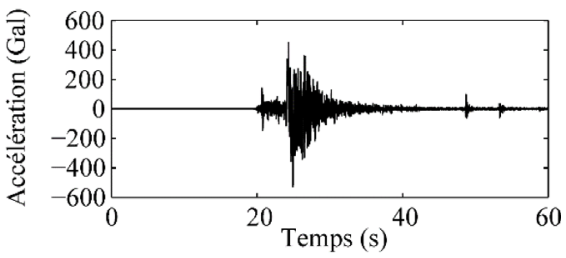

d)

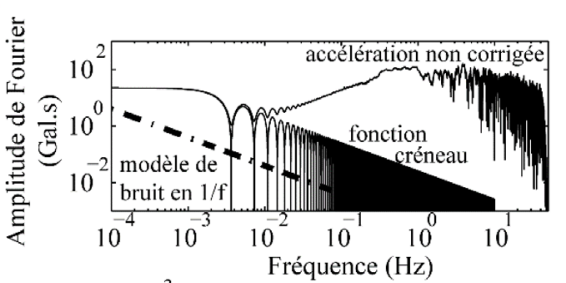

f)

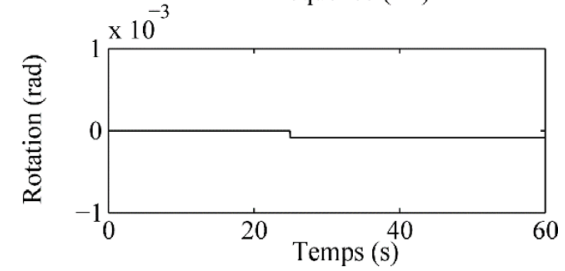

h)

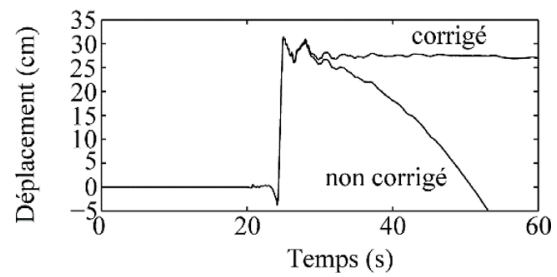

Fig. 11. $(a, b)$ Accélération non corrigée enregistrée à la base du barrage par les composantes EW et NS de l'accéléromètre pendant le séisme. (c, d) La superposition dans le domaine de Fourier des accélérations non corrigées et de fonctions créneau, d'amplitudes A $=-0,06952$ Gal et $\mathrm{A}=-0,08162 \mathrm{Gal}$ et de temps de départ $t_{s}=20,84 \mathrm{~s}$ et $t_{s}=24,96 \mathrm{~s}$ pour les composantes EW et NS, présente de très bonnes similitudes à basse fréquence. Le bruit en 1/f estimé indépendamment est bien au-dessous de l'amplitude de Fourier du signal : le contenu basse fréquence de l'accélérogramme est dominé par la rotation permanente. (e, f) Rotations permanentes estimées pour les composantes EW et NS à partir des fonctions créneau déterminées en c et $\mathrm{d} .(\mathrm{g}, \mathrm{h})$ Déplacements corrigé et non corrigé. Le déplacement corrigé est obtenu par soustraction des fonctions créneau des accélérogrammes non corrigés, avant d'intégrer deux fois ces accélérogrammes corrigés. Les déplacements sont stables après le séisme. Reproduit de Javelaud et al. (2012).

Fig. 11. (a, b) Uncorrected acceleration time histories recorded within the low inspection gallery by the strong-motion seismometer's EW and NS components during the main shock. (c, d) Superposition in the frequency domain of the uncorrected acceleration records and step functions, of respective amplitudes $A=-0.06952 \mathrm{Gal}$ and $A=-0.08162 \mathrm{Gal}$ and starting time $t_{s}=20.84 \mathrm{~s}$ and $t_{s}=24.96 \mathrm{~s}$ for the EW and NS components, shows extremely good match at low frequencies. The 1/f noise models estimated independently are well below the signals' Fourier amplitudes: the low frequency contents of the main shock records are therefore controlled by the permanent tilt information. (e, f) Permanent rotation estimated for the EW and NS components from the step functions derived in $c$ and $d .(g, h)$ Uncorrected and corrected displacement time histories. The corrected displacement time histories are obtained by subtracting the step functions from the uncorrected acceleration records, then double integrating the corrected acceleration time histories. The displacements are stable after the earthquake. From Javelaud et al. (2012).

déplacements obtenus par les deux méthodes sont presque identiques. Quand la distance entre les deux instruments augmente, on observe une bonne cohérence entre les déplacements obtenus par les deux méthodes. Ils mettent en évidence le déplacement du bloc supérieur vers le Nord-Ouest lors du jeu de la faille inverse.

La justesse des résultats obtenus a été confirmée par l'application de cette méthode à trois autres tremblements de terre, à savoir de Niigata-ken Chuetsu en 2004 (Javelaud et Morikawa, 2013 ; Javelaud et al., 2005, 2006, 2011b ; Ohtake, 2006 ; Ohtake et Ohmachi, 2007), de Noto Hanto en 2007
(Inoue et al., 2007 ; Murakami, 2008) et d'Iwate-Miyagi Nairuku en 2008 (Furukawa, 2011 ; Furukawa et al., 2010a, 2010b).

\subsection{Limites de la méthode}

Les limitations de la méthode sont de plusieurs types.

Tout d'abord de type instrumental. En effet, un bruit interne au capteur en $1 /$ f fort à basse fréquence rend la détermination du déplacement impossible. Cette problématique est actuellement en cours de résolution grâce à la fabrication et mise en place d'instruments avec un bruit interne toujours plus faible. 


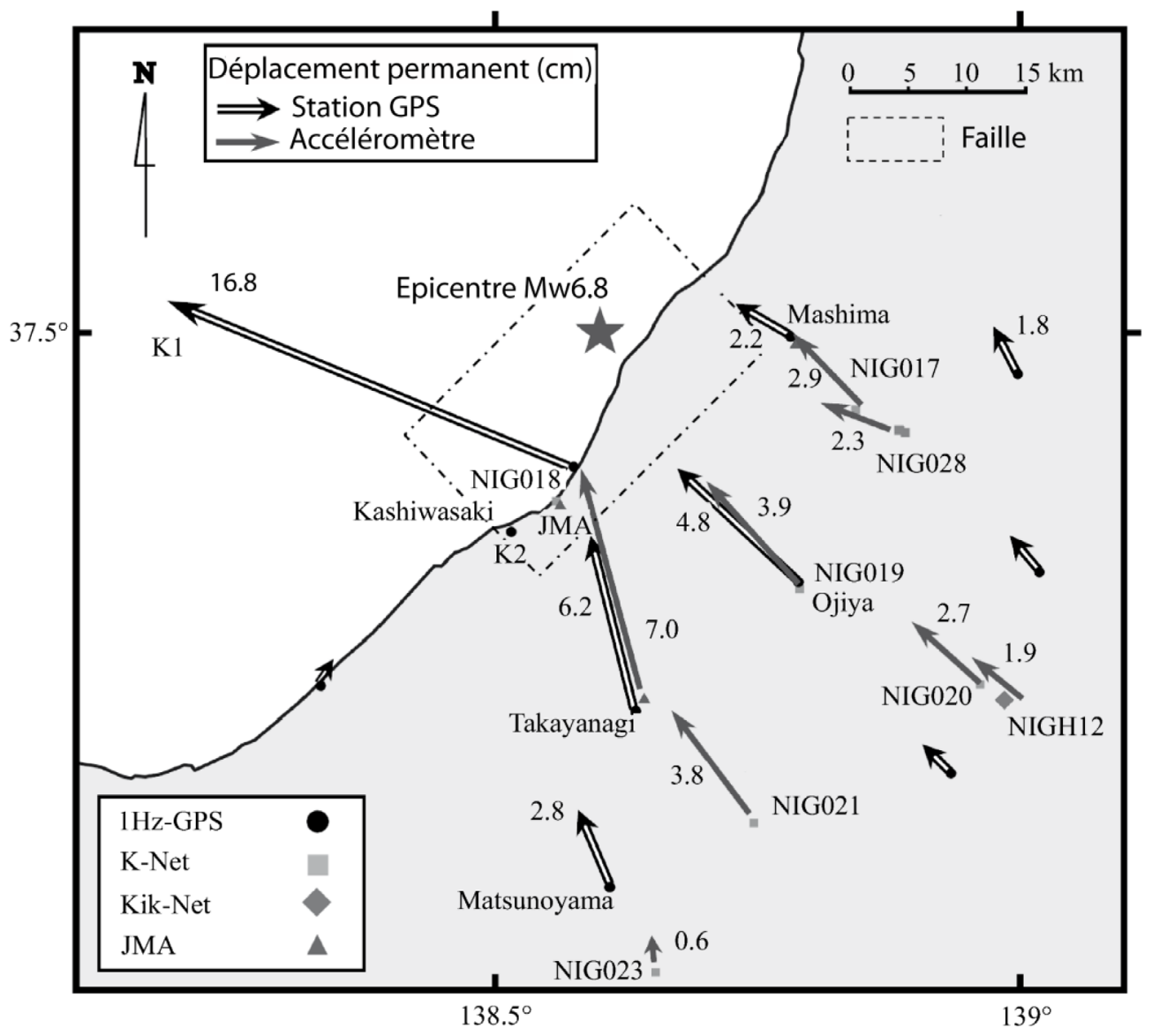

Fig. 12. Comparaison des déplacements permanents obtenus à partir d'accéléromètres et de stations GPS dans la zone épicentrale du séisme de Niigata-ken Chuetsu-Oki, Japon. Reproduit de Javelaud et al. (2011a).

Fig. 12. Comparison of coseismic residual displacement obtained from strong-motion seismometers and surrounding GPS stations during the 2007 Niigata-ken Chuetsu-Oki earthquake. From Javelaud et al. (2011a).

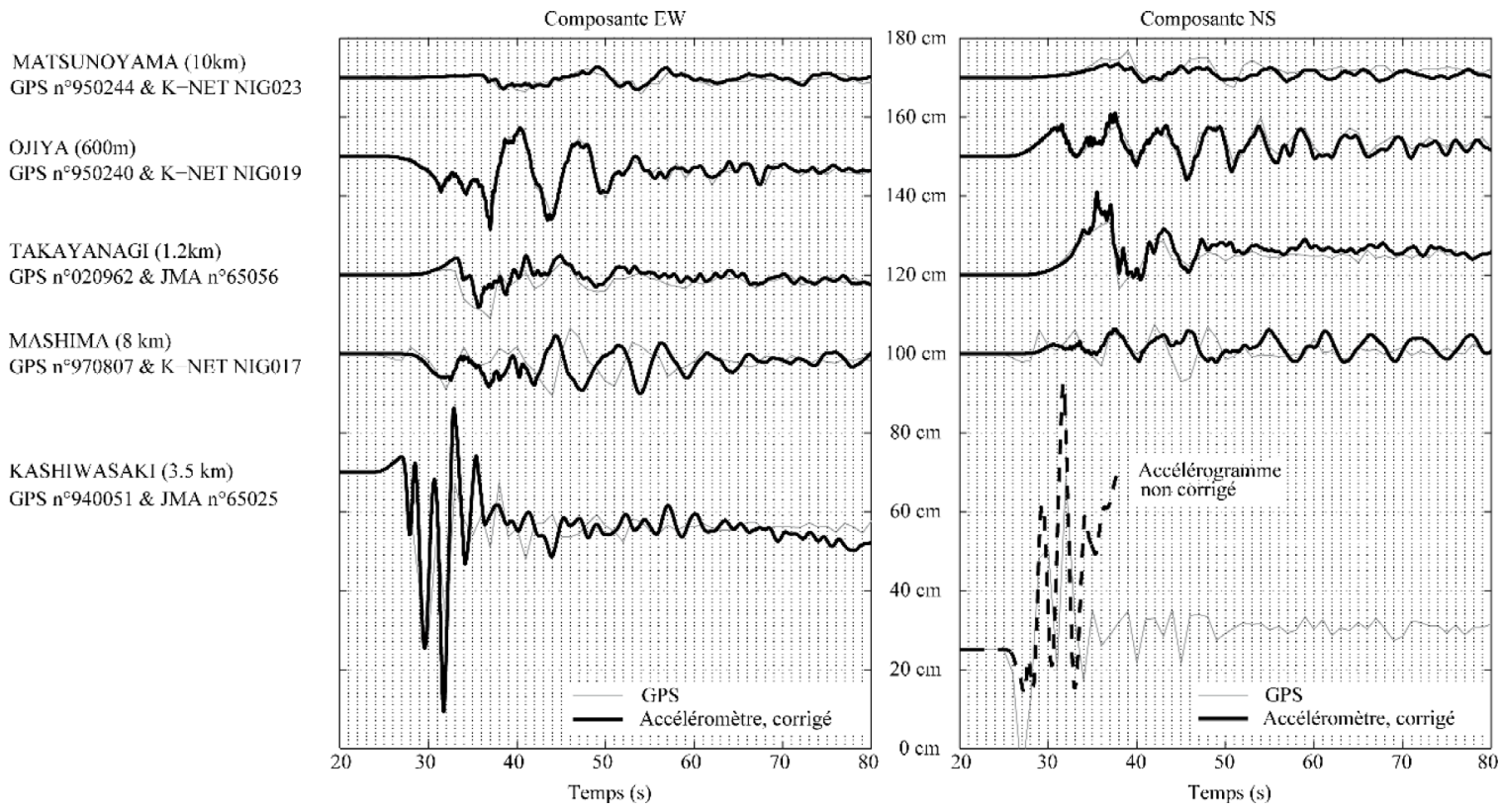

Fig. 13. Comparaison des déplacements obtenus à partir des enregistrements accélérométriques avec les données GPS 1-Hz pendant le séisme de Niigata-ken Chuetsu-Oki (2007, Japon). Reproduit de Javelaud et al. (2011a).

Fig. 13. Accelerometer displacement time series versus nearby 1-Hz GPS data recorded during the 2007 Niigata-ken Chuetsu-Oki earthquake, Japan. From Javelaud et al. (2011a). 


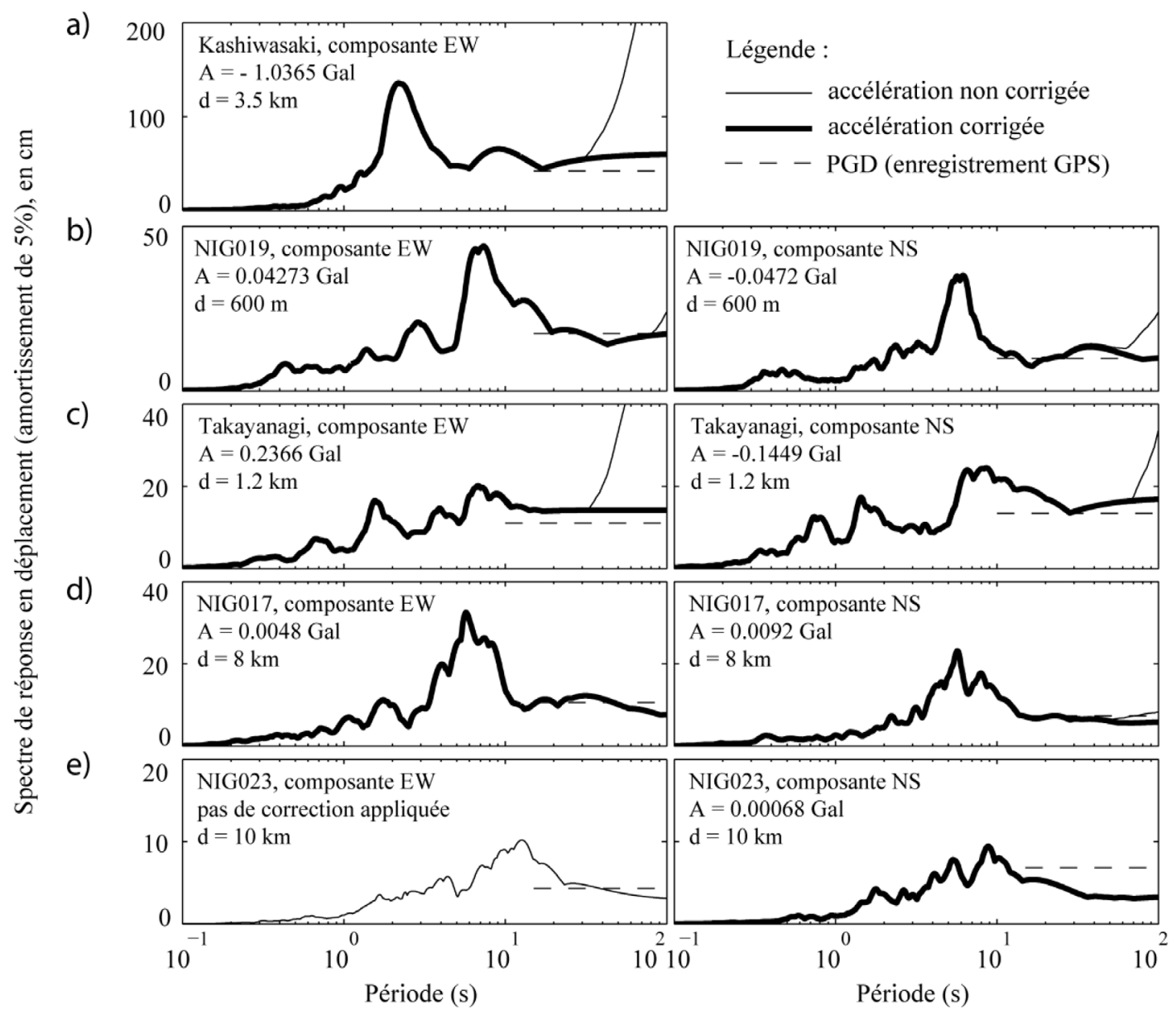

Fig. 14. Spectre de réponse en déplacement calculé à partir des accélérogrammes non corrigés et corrigés, ainsi que déplacement maximum obtenu à partir de la station GPS 1-Hz la plus proche à Kashiwasaki, Ojiya NIG019, Takayanagi, Mashima NIG017 and Matsunoyama NIG023. $A$ est l'amplitude de la rotation permanente soustraite de chaque accélérogramme non corrigé, et $d$ la distance séparant les accéléromètres de la station GPS la plus proche. Reproduit de Javelaud et Morikawa (2013).

Fig. 14. Displacement response spectra calculated from uncorrected and corrected acceleration time histories, together with the Peak Ground Displacement obtained from closely spaced 1-Hz GPS records at Kashiwasaki, Ojiya NIG019, Takayanagi, Mashima NIG017 and Matsunoyama NIG023. $A$ is the amplitude of the residual tilt removed from each uncorrected acceleration record, and d the distance from each strong-motion seismometer to its nearest GPS station. From Javelaud et Morikawa (2013).

Ensuite, la méthode nécessite une durée minimale des enregistrements. À titre d'exemple, les réseaux japonais K-Net et Kik-Net (NIED, 2014) fournissent des enregistrements de $300 \mathrm{~s}$ enregistrés avec une fréquence d'échantillonnage de $100 \mathrm{~Hz}$ ou $200 \mathrm{~Hz}$. Leur utilisation donne de bons résultats.

Une autre limitation est l'effet de la rotation pendant la durée du séisme. En zone épicentrale, soustraire la rotation permanente des accélérogrammes est nécessaire pour obtenir des déplacements réalistes et stables après le séisme. Cependant, au cœur de la zone épicentrale lors des séismes forts, cette hypothèse n'est plus forcément valide (Furukawa et al., 2010b ; Javelaud et al., 2011a) et la rotation pendant la durée du séisme doit être prise en compte.

De plus, la présence de multiples créneaux au sein d'un même accélérogramme génère plusieurs sauts de la ligne de base de l'accélérogramme qui ne peuvent actuellement pas être corrigés. Ces multiples créneaux peuvent être dus à l'enregistrement de plusieurs séismes et répliques, ou de plusieurs pulses comme observé à Kashiwasaki (composante NS) lors du séisme de Niigata-ken Chuetsu-Oki (2007).

\section{Applications pratiques pour le dimensionnement de structures}

Les accélérogrammes corrigés selon la méthode présentée aux Sections 4.2 et 4.3 peuvent être utilisés pour améliorer le dimensionnement et la justification de structures de génie civil. Ce peut être directement dans le domaine temporel pour des calculs sismiques transitoires ou bien sous la forme d'un spectre de réponse en accélération ou en déplacement.

\subsection{Calculs sismiques transitoires}

L'accélération translationnelle, le déplacement translationnel ainsi que la rotation permanente de l'instrument ainsi calculés à partir d'accélérogrammes peuvent être utilisés pour des analyses dans le domaine temporel cherchant à appréhender le comportement d'ouvrages à proximité de failles. Ce peut être comme données d'entrée pour des simulations numériques ou pour des modélisations expérimentales. 


\subsection{Méthodes en déplacement}

Les spectres de réponse en déplacement calculés à partir des accélérogrammes non corrigés (traits fins) et corrigés (traits épais) des cinq stations situées au plus proche de l'épicentre lors du séisme de Niigata-ken Chuetsu-Oki (Fig. 13) sont présentés sur la Figure 14. Le déplacement maximum mesuré par la station GPS la plus proche de chaque accéléromètre est également reporté sur la Figure 14 à fréquence nulle. Il indique la valeur théorique, aux écarts près dus à la distance entre les deux capteurs, que le spectre de réponse en déplacement devrait atteindre à grande période. Cela permet de s'assurer de la réalité du spectre aux grandes périodes.

Ce cas d'étude particulier montre que la rotation permanente est un facteur fondamental qui contrôle la partie grande période du spectre de réponse en déplacement (Fig. 14). Il est possible d'obtenir un spectre de réponse en déplacement réaliste sur une très large plage de périodes en supprimant la rotation permanente qui perturbe les accélérogrammes.

Par ailleurs, il apparait ici que soustraire la rotation permanente des accélérogrammes ne modifie pas de façon significative le spectre de réponse en déplacement jusqu'à des périodes d'environ $20 \mathrm{~s}$, ce qui est cohérent avec les études antérieures (Akkar et Boore, 2009 ; Paolucci et al., 2008 ; Wang et al., 2007).

\section{Conclusions}

Cette contribution présente une méthode originale qui permet de calculer le déplacement ainsi que des rotations permanentes réalistes à partir d'accélérogrammes enregistrés en zone épicentrale lors de forts séismes. L'originalité de l'approche proposée réside en une analyse détaillée des accéléromètres et de leurs différentes sources de bruit, ainsi que du contenu basse fréquence du signal enregistré dans le domaine de Fourier.

Une des limitations instrumentales à l'application de cette méthode est le bruit interne en $1 / \mathrm{f}$ que contiennent les accélérogrammes à basse fréquence. Cette problématique est en cours de résolution par la fabrication et le déploiement d'instruments de meilleure qualité ayant un bruit interne de plus en plus faible. Ceci contribue et contribuera à faciliter l'application de cette méthode à un nombre d'instruments et d'enregistrements toujours plus grand.

Remerciements. À mes Maîtres : les professeurs F. Masrouri et J.-M. Montel de l'École Nationale Supérieure de Géologie de Nancy qui ont guidé mes premiers pas en géotechnique et en géologie, et T. Ohmachi et H. Morikawa du Tokyo Institute of Technology (Japon) qui m’ont formé en sismologie.

\section{Références}

Akkar S, Boore DM. 2009. On baseline corrections and uncertainty in response spectra for baseline variations commonly encountered in digital accelerograph records. Bull Seismol Soc Am 99: 1671-1690.

Bock Y, Agnew D, Fang P, et al. 1993. Detection of crustal deformation from the Landers earthquake sequence using continuous geodetic measurements. Nature 361: 337-340.
Boore DM. 2001. Effect of baseline corrections on displacements and response spectra for several recordings of the 1999 Chi-Chi, Taiwan, earthquake. Bull Seismol Soc Am 91: 1199-1211.

Boore DM, Bommer JJ. 2005. Processing of strong-motion accelerograms: needs, options and consequences. Soil Dyn Earthquake Eng 25: 93-115.

Dotsenko SF, Soloviev SL. 1995. On the role of residual shifts of ocean-bottom in tsunami generation underwater earthquakes. Okeanologiya 51: 25-31.

Furukawa A. 2011. Relation between observed ground displacement in the near-field and ground displacement based on Seismic Deformation Method. Master thesis, Tokyo Institute of Technology.

Furukawa A, Inoue S, Ohmachi T. 2010a. Verification of a quantitative method to estimate coseismic displacements in near-field from strong-motion accelerographs. In: Japan Society of Civil Engineers, 2010 Annual Meeting, I-326 (en japonais).

Furukawa A, Ohmachi T, Inoue S. 2010b. Need to improve the Seismic Deformation Method in application to near-field earthquakes. In: Proceedings of the Second Symposium on Disaster Mitigation of Lifelines considering System Interactions, pp. 64-69.

Graizer VM. 2010. Strong motion recordings and residual displacements: what are we actually recording in strong motion seismology? Seism Res Lett 81: 635-639.

Inoue S, Murakami Y, Ohmachi T. 2007. Evaluation of the near-field ground displacements based on numerical simulation, strong motion records and GPS records. In: Proceedings of the 6th Annual Meeting of Japan Association for Earthquake Engineering, pp. 218-219 (en japonais).

Intergovernmental Oceanographic Commission. 2013. Tsunami glossary. Technical series, 85.

Iwan WD, Moser MA, Peng CY. 1985. Some observations on strong motion earthquake measurement using a digital accelerograph. Bull Seismol Soc Am 75: 1225-1246.

Javelaud EH. 2013. Near-field displacement and rotation estimated by quantitative analysis in the frequency domain using zero padded strong-motion accelerograms. Doctor thesis. Tokyo Institute of Technology (http://t2r2.star.titech.ac.jp/rrws/file/CTT100691251/ ATD100000413/, dernier accès le 19 janvier 2016).

Javelaud EH, Morikawa H. 2013. Reliable displacement response spectra at long periods in the near-field of large earthquakes. Bull Seismol Soc Am 103: 2534-2539.

Javelaud EH, Kubo G, Ohmachi T, Inoue S. 2005. Coseismic ground displacement due to the 2004 Niigata-ken Chuetsu earthquake, Japan. In: Proceedings of the 4th Annual Meeting of Japan Association for Earthquake Engineering, pp. 312-313.

Javelaud EH, Ohmachi T, Inoue S. 2006. A quantitative method to estimate the coseismic residual tilt from strong-motion records. In: Proceedings of the 30th Annual Meeting of San-daigakuin, pp. 5-6.

Javelaud EH, Ohmachi T, Inoue S. 2011a. A quantitative approach for estimating coseismic displacements in the near field from strongmotion accelerographs. Bull Seismol Soc Am 101: 1182-1198.

Javelaud EH, Inoue S, Furukawa A, Ohmachi T. 2011b. Evaluation of coseismic displacements in the near-field from strong-motion seismometers. Analysis of four recent large earthquakes. In: 8 th International Conference on Urban Earthquake Engineering (8CUEE), Tokyo, Japan, pp. 189-192.

Javelaud EH, Ohmachi T, Inoue S. 2012. Estimating small permanent rotation from strong-motion records: what is comparison with external measurements telling us? Bull Seismol Soc Am 102: 2257-2263.

JCOLD (Japan Commission on Large Dams). 2002. Acceleration records on dams and foundations No. 2 (CD-ROM version). 
Japan Society of Civil Engineers. 2000. Earthquake resistant design codes in Japan.

Kinoshita S. 1998. Kyoshin-Net (K-Net). Seism Res Lett 69: 309-332.

Levin BW, Nosov MA. 2008. Physics of tsunamis. Springer, 327 p.

Massonnet D, Rossi M, Carmona C, et al. 1993. The displacement field of the Landers earthquake mapped by radar interferometry. Nature 364: 138-142.

McComb HE, Ruge AC, Neumann F. 1943. The determination of true ground motion by integration of strong-motion records: a symposium. Bull Seismol Soc Am 33: 1-63.

Murakami Y. 2008. Evaluation of near field displacements. Master thesis, Tokyo Institute of Technology.

National Research Institute for Earth Science and Disaster Prevention, Japan (NIED). Seismograph Network Portal (http://www.seis.bosai. go.jp/seis-portal/, dernier accès le 27 août 2014).

NIED. 2000. Fundamentals of Strong Motion, CD version.

Ohmachi T, Kojima N, Murakami A, Komaba N. 2003. Near field effects of hidden seismic faulting on a concrete dam. J Nat Disaster Sci 25: 7-15.

Ohtake K. 2006. Near-field earthquake displacements of the nonliquefiable ground relevant to damage to buried pipelines. Doctor thesis. Tokyo Institute of Technology.
Ohtake K, Ohmachi T. 2007. Near-field earthquake displacements of the non-liquefiable ground relevant to damage to buried pipelines. In: 5th AWWARF/JWWA Water System Seismic Conference.

Paolucci R, Rovelli A, Faccioli E, et al. 2008. On the reliability of long-period response spectral ordinates from digital accelerograms. Earthquake Eng Struct Dyn 37: 697-710.

Park SW, Ghasemi H, Shen J, Somerville PG, Yen WP, Yashinsky M. 2004. Simulation of the seismic performance of the Bolu Viaduct subjected to near-field ground motions. Earthquake Eng Struct Dyn 33: $1249-1270$.

Pillet R, Virieux J. 2007. The effects of seismic rotations on inertial sensors. Geophys J Int 171: 1314-1323.

Trifunac MD. 1971. Zero baseline correction of strong-motion accelerograms. Bull Seismol Soc Am 61: 1201-1211.

Trifunac MD, Udwadia FE, Brady AG. 1973. Analysis of errors in digitized strong-motion accelerograms. Bull Seismol Soc Am 63: $157-187$

Wang GQ, Boore DM, Tang G, Zhou X. 2007. Comparisons of ground motions from colocated and closely spaced one-sample-per-second global positioning system and accelerograph recordings of the 2003 M 6.5 San Simeon, California, Earthquake in the Parkfield region. Bull Seismol Soc Am 97: 76-90.

Cite this article as: Emmanuel Javelaud. Déplacements et rotations du sol lors de forts séismes à proximité de failles actives : apports des capteurs accélérométriques. Rev. Fr. Geotech. 2016, 146, 3. 